\title{
Large granular lymphocytic leukaemia complicated with histiocytic sarcoma in a dog
}

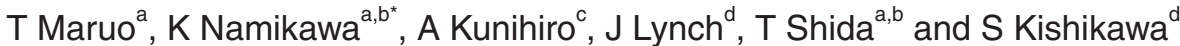

\begin{abstract}
A 10-year-old castrated male Golden retriever, weighing $36.3 \mathrm{~kg}$ was referred for evaluation owing to a decline in general condition. Findings from the complete blood count revealed a marked lymphocytosis $(113000 / \mu l)$. Examination of Wright-Giemsa-stained films of peripheral blood revealed the presence of large granular lymphocytes (LGL). Seventy-two per cent $(81360 / \mu l)$ of the lymphocytes were found to be $12-17 \mu \mathrm{m}$ in diameter, containing nuclei with mature clumped chromatin and abundant lightly basophilic cytoplasm with a variable number of fine azurophilic granules. Based on these findings this case was diagnosed as LGL leukaemia. As a result of multiple-agent chemotherapy, the markedly elevated levels of lymphocytes gradually decreased to $7500 / \mu l$ on day 122 and the patient maintained a good quality of life for the following 3 months. However, on around day 237 , a soft, raised, bosselated mass on the labial region was noted. The dog was diagnosed as having histiocytic sarcoma based on cytological and histological examination of the mass. Shortly after diagnosis, the dog developed sudden onset of central nervous system signs and died on day 270. A common outcome of canine LGL is the development of acute blast crisis or lymphoma. However, this case was notable for complication with histiocytic sarcoma from another origin.
\end{abstract}

Keywords: dog, histiocytic sarcoma, large granular lymphocytic leukaemia.

Maruo T, Namikawa K, Kunihiro A, Lynch J, Shida T, Kishikawa S Large granular lymphocytic leukaemia complicated with histiocytic sarcoma in a dog. Journal of the South African Veterinary Association (2009) 80(4): 261-263 (En.). Veterinary Teaching Hospital, Azabu University, 1-17-71 Fuchinobe, Sagamihara, Kanagawa 229-8501, Japan

\section{INTRODUCTION}

Canine large granular lymphocytic (LGL) leukaemia is a chronic lymphocytic leukaemia (CLL), characterised by a neoplastic lymphocyte population originating in NK or cytotoxic T cell proliferation clonally consists of large cells with abundant, lightly basophilic cytoplasm with azurophilic granules ${ }^{6,7,12-14,16}$. Primarily large-breed dogs, such as the Golden retriever, German shepherd and Siberian husky are affected and the disease tends to occur in older dogs of 8-13 years ${ }^{7,13,16}$. LGL leukaemia is usually a non-aggressive disease and thus may remain dormant for long periods during the illness ${ }^{6,7,16}$.

Conversely, histiocytic sarcoma is an aggressive, commonly metastatic systemic neoplasm of atypical histiocytes originating

aVeterinary Teaching Hospital, Azabu University, 1-17-71 Fuchinobe, Sagamihara, Kanagawa 229-8501, Japan.

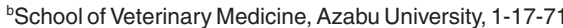
Fuchinobe, Sagamihara, Kanagawa 229-8501, Japan.

'Momo Animal Clinic 1-8-9 Nakamachidai, Tsuzukiku, Yokohama, Kanagawa 224-0041, Japan.

${ }^{d}$ School of Life and Environment Science, Azabu University, 1-17-71 Fuchinobe, Sagamihara, Kanagawa 229 8501, Japan.

*Author for correspondence.

E-mail: namikawa@ azabu-u.ac.jp

Received: June 2009. Accepted: November 2009. in dendritic cells, affecting primarily the Bernese mountain dog, Rottweiler, Golden retriever and Flat-coated retriever, with multi-organ involvement possible $e^{1-3,10}$. Clinical findings commonly depend on the lesion site, respiratory abnormalities, neurological signs and anaemia. Untreated tumours have an aggressive clinical course which is invariably fatal ${ }^{1-3,9-11}$.

In this clinical communication we present a canine case of LGL in which a good therapeutic response was achieved after immunosuppressive therapy but which subsequently became complicated with histiocytic sarcoma.

\section{CASE HISTORY}

A 10-year-old castrated male Golden retriever, weighing $36.3 \mathrm{~kg}$, was referred to the Veterinary Teaching Hospital of Azabu University, Japan, for evaluation, owing to a decline in general condition that was accompanied by gradual weight loss, weakness and anorexia observed over a period of about 1 year. Although physical examination did not identify lymphadenopathy, abdominal radiographs revealed evidence of splenomegaly. The complete blood count (CBC) revealed marked leukocytosis $(135000 / \mu \mathrm{l})$, including lymphocytosis $(113000 / \mu$ l) (Fig. 1). An examination of Wright-Giemsa-stained films of peripheral blood revealed the presence of large granular lymphocytes (Fig. 2A); $72 \%(81360 / \mu l)$ of lymphocytes were found to be $12-17 \mu \mathrm{m}$ in diameter, compared with normal lymphocytes ${ }^{5}$ which are 6-9 $\mu \mathrm{m}$, and contained nuclei with mature clumped chromatin and abundant, lightly basophilic cytoplasm with a variable number of fine azurophilic granules. CBC further revealed mild anaemia but as reticulocytes were not increased, this observation was suggested to be a result of depression of haemopoiesis. Aspiration and core biopsy of bone marrow revealed mature lymphocytes containing cytoplasmic granules and clumped nuclear chromatin similar to those observed with light microscopy in the peripheral blood, accounting for about half of all nucleated cells $(47.5 \%)$. Overall, immature lymphoblasts were not found and myeloid cells and erythroid cells were observed at a low level of $30 \%$ and $17 \%$, respectively. Ultrastructural examination revealed fine heterochromatin granules in the nuclear membrane, which were also present diffusely in the nuclei and seemed to be of mature shape. Elliptical granules $0.4-0.8 \mu \mathrm{m}$ in diameter with an electron-dense core were present in the cytoplasm, a result similar to that observed by light microscopic investigation (Fig. 2B). LGL proliferative disorders of dogs can be either neoplastic or non-neoplastic ${ }^{7,16}$. Non-neoplastic LGL lymphocytosis can occur secondary to chronic Ehrlichia canis infection ${ }^{4}$. Although canine ehrlichiosis is not found in Japan, immunoglobulin IgG antibody titres for the agent of canine ehrlichiosis causing reactive LGL lymphocytosis were nevertheless investigated. As expected, the results were negative and ruled LGL out as a secondary disorder. This case was therefore diagnosed as LGL leukaemia classified as chronic lymphocytic leukaemia.

Despite the fact that the dog did not appear to be compromised, observed clinical signs included lethargy and weight loss. To reduce the tumour burden 


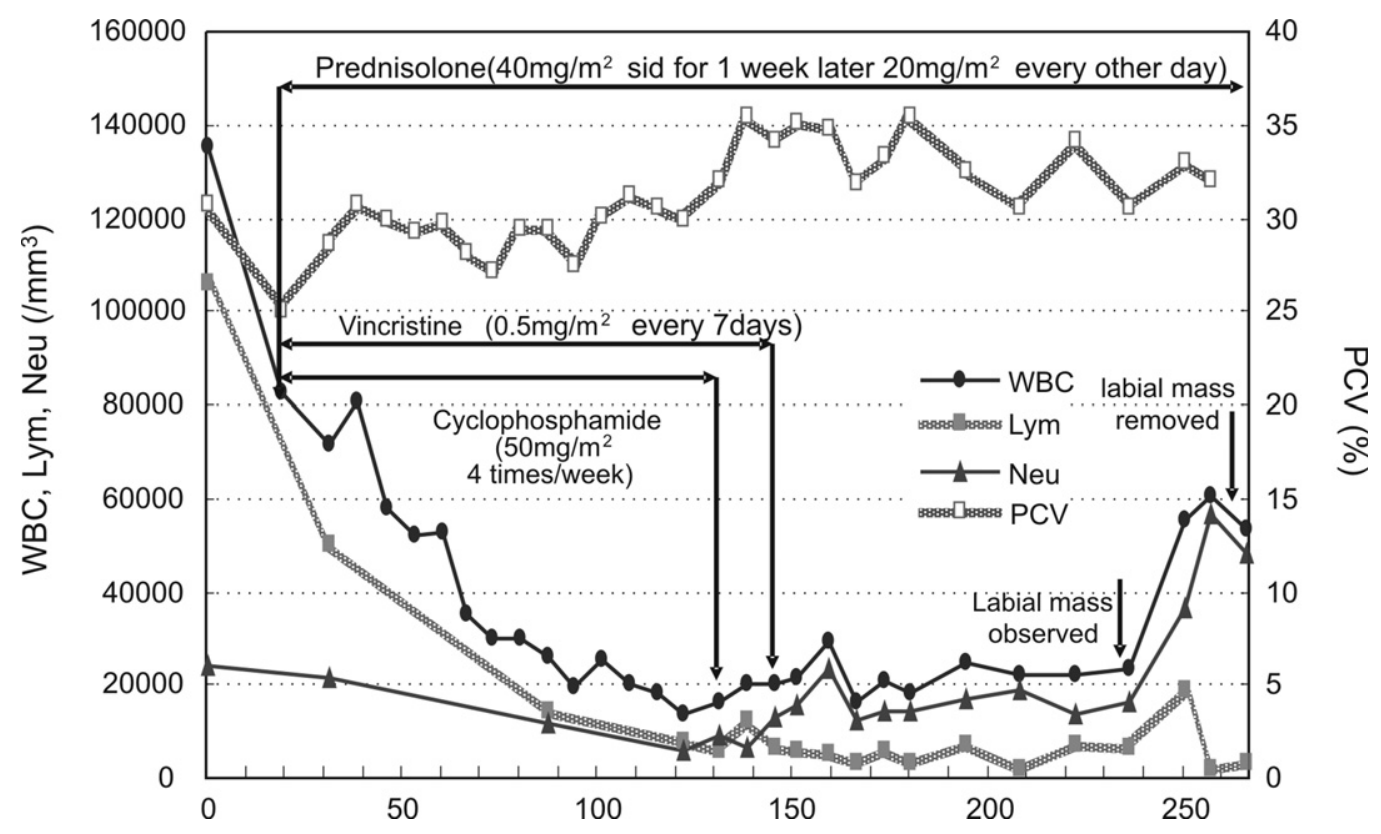

Fig. 1: Clinical course of case. White blood cell, lymphocyte and neutrophil counts, packed cell volume and treatment employed. Lymphocytosis and anaemia gradually improved with the chemotherapy until day 122. Subsequently, a mass on the labial region was observed on around day 237 and removed on day 267. WBC: white blood cell; Lym: lymphocytes; Neu: neutrophil; PVC: packed cell volume.

for as long as possible, multiple agent chemotherapy with cyclophosphamide (Endoxan ${ }^{\circledast}$, Shionogi Co.) was administered at $50 \mathrm{mg} / \mathrm{m}^{2}$ for 4 consecutive days followed by 3 consecutive days without treatment days for 14 weeks (Fig. 1). Vincristine (Oncovin ${ }^{\circledR}$, Nippon Kayaku Co.) at $0.5 \mathrm{mg} / \mathrm{m}^{2}$ once a week for 16 weeks and prednisolone (Prednisolone ${ }^{\circledR}$, Sanwa Kagaku Kenkyusho Co.) at $20-40 \mathrm{mg} / \mathrm{m}^{2}$ once a day to every other day were also administered. As a result of these treatments, the markedly elevated levels of lymphocytes gradually decreased to $7500 / \mu$ l on day 122 and remained at this level after that; PCV values increased from day 20 to day 139 to $25 \%$ and $36 \%$, respectively, and general condition remained good without tumour lysis syndrome from that point onwards.

The patient's physical condition had been good since around day 122 after discontinuing the cyclophosphamide and vincristine treatment and continuing prednisolone alone for 3 months. However, on around day 237 when PCV decreased, a soft, raised, bosselated mass on the labial region was noted. Because the mass rapidly enlarged at around 251 days, it was resected under general anaesthesia on day 267. The mass was examined cytologically and histologically. Impression smears stained with WrightGiemsa and sections stained with haematoxylin and eosin (Fig. 3) revealed numerous histiocytes that appeared necrotic with mitotic figures, multinucleated tumour giant cells and erythrophagocytosis. Based on these findings, the dog was diagnosed as having histiocytic sarcoma. Immediately after the diagnosis was made, the dog developed sudden onset of central nervous system (CNS) signs and died on day 270 . A necropsy was not performed.
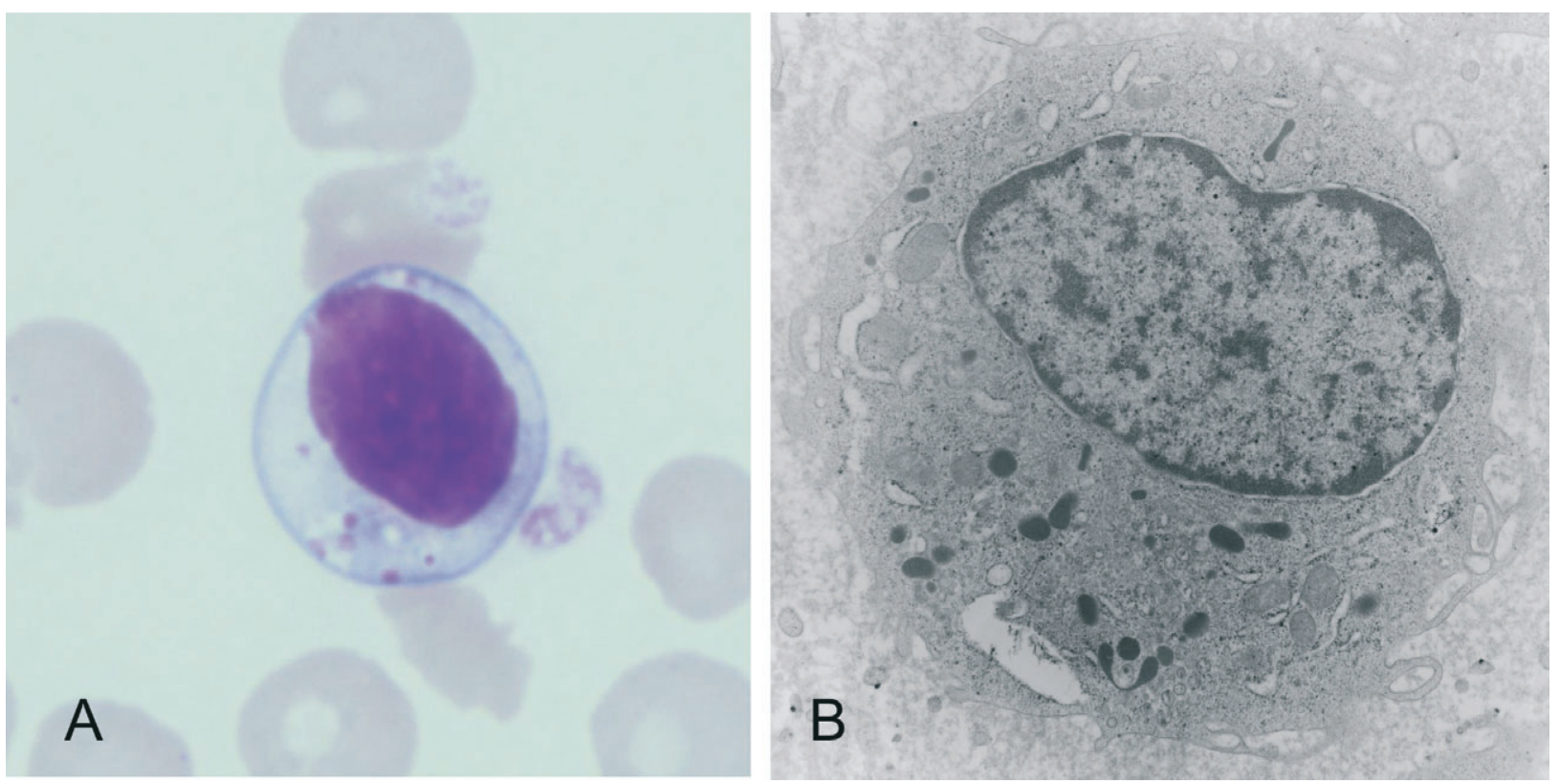

Fig. 2: Large granular lymphocyte of peripheral blood. A, Photomicrograph; lymphocytes have nuclei with mature clumped chromatin and a pale cytoplasm that contains granules. B, Electron micrograph; fine heterochromatin granules are in the nuclear membrane and also diffusely in the nuclei, the cytoplasm has abundant volume, a rough-surfaced endoplasmic reticulum and some rounded granules with electron-dense cores. 


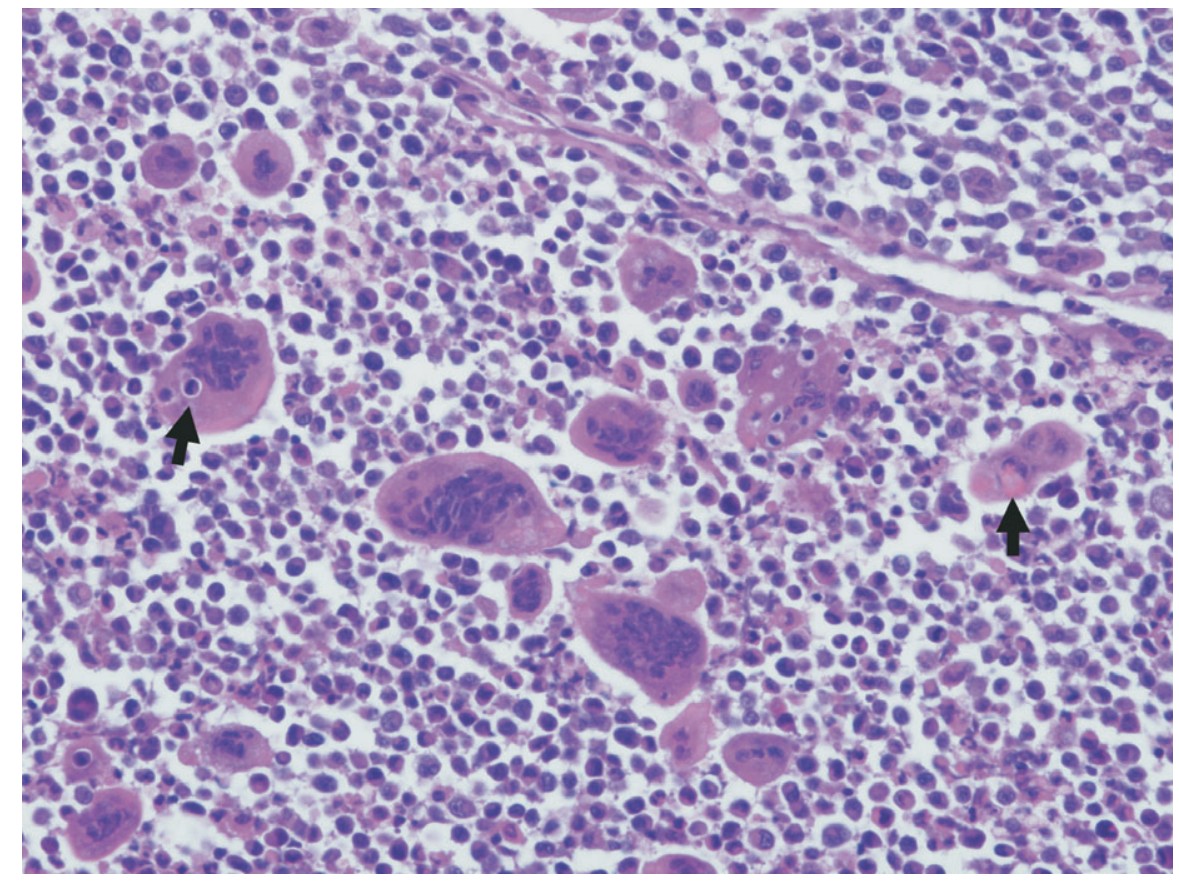

Fig. 3: Histology of labial mass (H\&E stain). There are abundant multinucleated giant cells and numerous immature anisocytic histiocytes. Some giant cells contain intact red blood cells and mononuclear cells (arrows). Some histocytes reveal karyomitosis.

\section{DISCUSSION}

As immunophenotyping of peripheral lymphocytes was not carried out it was not possible to distinguish the subtype as T-LGL or NK-LGL. The case was therefore diagnosed as LGL leukaemia, classified as chronic lymphocytic leukaemia, based on the $\mathrm{CBC}$, light microscopic and ultrastructural findings. Because LGL leukaemia is often indolent, treatment is not always required. As the general condition of this case gradually worsened, multi-immunosuppressive drugs were employed in a combination to which canine LGL is known to respond well. A common outcome of canine LGL is the development of acute blast crisis or lymphoma $^{8,15}$. However, this case involved the sudden development of histiocytic sarcoma, with the possibility that the mass might have metastasised to the CNS. It was not possible to determine why 2 malignant diseases had occurred in $1 \mathrm{dog}$. This is the 1st reported case in a dog in which canine LGL leukaemia was complicated with histiocytic sarcoma from another origin.

\section{REFERENCES}

1. Carioto L 1997 Malignant histiocytosis in a Bernese mountain dog presenting as a mandibular mass. Canadia Veterinary Journal 38: 105-107

2. Chandra A M S, Ginn P E 1999 Primary malignant histiocytosis of the brain in a dog. Journal of Comparable Pathology 121: 77-82

3. Hayden D W, Waters D J, Burke B A, Manivel J C 1993 Disseminated malignant histiocytosis in a Golden retriever: clinicopathologic, ultrastructural, and immunohistochemical findings. Veterinary Pathology 30: 256-264

4. Heeb H L, Wilkerson M J, Chun R, Ganta R R 2003 Large granular lymphocytosis, lymphocyte subset inversion, thrombocytopenia, dysproteinemia, and positive
Ehrlichia serology in a dog. Journal of the American Animal Hospital Association 39: 379-384

5. Jain N C 1993 The lymphocytes and plasma cells. In: Jain N C (ed.) Essentials of veterinary hematology. Lea \& Febiger, Philadelphia: 278-294

6. Lau K W M, Kruth S A, Thorn C E, Vernau W, Moore P 1999 Large granular lymphocytic leukemia in a mixed breed dog. Canadian Veterinary Journal 40: 725-728

7. McDonough S P, Moore P F 2000 Clinical, hematologic, and immunophenotypic characterization of canine large granular lymphocytosis. Veterinary Pathology 37: 637-646

8. Mylonakis M E, Koutinas A F, Papaioannou N, Lekkas S 2007 Acute tumor lysis syndrome in a dog with B-cell multicentric lymphoma. Australian Veterinary Journal 85: 206-208

9. Ramirez S, Douglass J P, Robertson I D 2002 Ultrasonographic features of canine abdominal malignant histiocytosis. Veterinary Radiology \& Ultrasound 43: 167-170

10. Ramsey I K, McKay J S, Rudorf H, Dobson J M 1996 Malignant histiocytosis in three Bernese mountain dogs. Veterinary Record 138: $440-444$

11. Skorupski K A, Clifford C A, Paoloni M C, Lara-Garcia A, Barber L, Kent M S, LeBlanc A K, Sabhlok A, Mauldin E A, Shofer F S, Couto C G, Srenmo K U 2007 CCNU for the treatment of dogs with histiocytic sarcoma. Journal of Veterinary Internal Medicine 21: 121-126

12. Snead E C 2007 Large granular intestinal lymphosarcoma and leukemia in a dog. Canadian Veterinary Journal 48: 848-851

13. Takahashi T, Otani I, Okuda M, Inoue M, Ito K, Sakai M, Koie H, Yamaya Y, Watari T, Sato T, Kanayama K, Tokuriki M 2007 Malignant transformation of T-cell large granular lymphocyte leukemia in a dog. Journal of Veterinary Medical Science 69: 677-681

14. Vernau W, Moore P F 1999 An immunophenotypic study of canine leukemias and preliminary assessment of clonality by polymerase chain reaction. Veterinary Immunology and Immunopathology 69: 145-164

15. Vickery K R, Thamm D H 2007 Successful treatment of acute tumor lysis syndrome in a dog with multicentric lymphoma. Journal of Veterinary Internal Medicine. 21: 1401-1404

16. Workman H C, Vernau W 2003 Chronic lymphocytic leukemia in dogs and cats: the veterinary perspective. Veterinary Clinics of North America: Small Animal Practice 33: 1379-1399 